\title{
Development of Cerebral Gray and White Matter Injury and Cerebral Inflammation over Time after Inflammatory Perinatal Asphyxia
}

\author{
Hilde J.C. Bonestroo ${ }^{a} \quad$ Cobi J. Heijnen ${ }^{a, c} \quad$ Floris Groenendaal $^{b} \quad$ Frank van Bel $^{b}$ \\ Cora H. Nijboer ${ }^{a}$

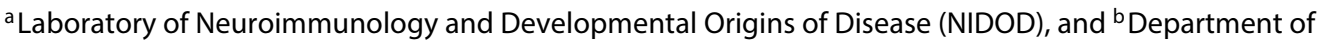 \\ Neonatology, University Medical Center Utrecht, Utrecht, The Netherlands; ' $\mathrm{D}$ Department of Symptom Research, \\ Division of Internal Medicine, the University of Texas MD Anderson Cancer Center, Houston, Tex., USA
}

\section{Key Words}

Neonatal brain injury · Inflammation · Lipopolysaccharide · Hypoxia-ischemia · Cytokines · Microglia · Neutrophils · White matter $\cdot$ Oligodendrocytes

\begin{abstract}
Antenatal inflammation is associated with increased severity of hypoxic-ischemic (HI) encephalopathy and adverse outcome in human neonates and experimental rodents. We investigated the effect of lipopolysaccharide (LPS) on the timing of HI-induced cerebral tissue loss and gray matter injury, white matter injury and integrity, and the cerebral inflammatory response. On postnatal day 9, mice underwent $\mathrm{HI}$ by unilateral carotid artery occlusion followed by systemic hypoxia which resulted in early neuronal damage (MAP2 loss) at $3 \mathrm{~h}$ that did not increase up to day 15. LPS injection $14 \mathrm{~h}$ before $\mathrm{HI}(\mathrm{LPS}+\mathrm{HI})$ significantly and gradually aggravated MAP2 loss from $3 \mathrm{~h}$ up to day 15, resulting in an acellular cystic lesion. LPS $+\mathrm{HI}$ increased white matter damage, reduced myelination in the corpus callosum and increased white matter fiber coherency in the cingulum. The number of oligodendrocytes throughout the lineage (Olig2-positive) was increased whereas more mature myelinating (CNPase-positive) oligodendrocytes were strongly decreased after LPS+HI. LPS $+\mathrm{HI}$ induced an increased and prolonged expression of cerebral cytokines/chemokines compared to HI. Addition-
\end{abstract}

ally, LPS+HI increased macrophage/microglia activation and influx of neutrophils in the brain compared to HI. This study demonstrates the sensitizing effect of LPS on neonatal HI brain injury for an extended time-frame up to 15 days postinsult. LPS before $\mathrm{HI}$ induced a gradual increase in gray and white matter deficits, including reduced numbers of more mature myelinating oligodendrocytes and a decrease in white matter integrity. Moreover, LPS+HI prolonged and intensified the cerebral inflammatory response, including cellular infiltration. In conclusion, as the timing of damage and/ or involved pathways are changed when $\mathrm{HI}$ is preceded by inflammation, experimental therapies might require modifications in the time window, dosage or combinations of therapies for efficacious neuroprotection. @ 2015 S. Karger AG, Basel

\section{Introduction}

Neonatal encephalopathy due to perinatal asphyxia continues to be an important cause of mortality and morbidity in the human neonate. Perinatal brain injury often results in severe and long-lasting disabilities, like motor deficits, seizures and severe cognitive, psychological and behavioral problems, which represent an enormous burden for the child, its parents and society [1-3]. Importantly, the risk of developing neonatal encephalopathy is

\section{KARGER 125}

C 2015 S. Karger AG, Basel

0378-5866/15/0371-0078\$39.50/0

E-Mail karger@karger.com

www.karger.com/dne
Cora H. Nijboer, $\mathrm{PhD}$

Laboratory of Neuroimmunology and Developmental Origins of Disease (NIDOD) University Medical Center Utrecht

HP KC03.068.0, PO Box 85500, 3508 GA Utrecht (The Netherlands)

E-Mail c.nijboer@umcutrecht.nl 
strongly increased after maternal intrapartum fever or other antenatal inflammatory conditions like chorioamnionitis [4-7]. Moreover, it is known that exposure of the fetal brain to a proinflammatory environment in utero negatively influences clinical outcome after asphyxia, including an increased risk of development of spastic cerebral palsy [8-11]. In an attempt to mirror this clinical situation, several animal models have been developed over the past decade. Most of these models use lipopolysaccharide (LPS), a structural component of most Gramnegative bacteria that binds to Toll-like receptor 4 , as the inflammatory stimulus [12]. To investigate the consequences of a proinflammatory environment for the neonatal brain, administration of LPS to the pregnant mother (either systemically or locally in the uterus) or direct administration of LPS to the newborn rodent (systemically or locally in the brain) have been tested [13]. With respect to studies on neonatal brain damage, LPS is often combined with hypoxia-ischemia (HI), the latter first described by Rice et al. [14]. The contribution of inflammation to HI brain damage is complex and conflicting results have been obtained when exploring the dose and time frame of LPS application before inducing HI. The general view is that inflammation before $\mathrm{HI}$ sensitizes the brain and thereby aggravates HI cerebral injury. However, the exact mechanisms underlying proinflammatory sensitization are still unclear [15-21]. To date, the exact timing of the sensitizing effect of LPS on the intensity of cerebral gray and white matter injury and the formation of an acellular cyst after $\mathrm{HI}$ have not been investigated in detail. Moreover, most studies only used loss of myelin basic protein (MBP) staining as a marker for white matter injury, whereas the effect of a proinflammatory environment in the context of $\mathrm{HI}$ on white matter integrity and on differentiation of oligodendrocytes remains largely unknown. In addition, it is of great importance to know the effect of combined exposure to LPS and HI on the degree, timing and duration of the cerebral inflammatory response, including the induction of proinflammatory or anti-inflammatory cytokines/chemokines, activation of microglia and influx of neutrophils and macrophages into the cerebral parenchyma. Closely mapping the development of injury and the cerebral inflammatory response in the LPS+HI model, which mimics the clinical situation in near-term neonates, is an essential step to allow exploration of future therapeutic strategies for the neonate facing perinatal asphyxia combined with a proinflammatory state.

The data within this paper show the precise timing of development of cerebral tissue loss, gray and white matter damage, and integrity in response to LPS+HI over a time window of $3 \mathrm{~h}$ up to day (D) 15 postinsult. In parallel, we determined the effect of LPS+HI on the number of oligodendrocytes throughout the lineage and more mature oligodendrocytes as well as on the cerebral inflammatory response and infiltration of inflammatory cells into the brain in the first 3 days postinsult.

\section{Materials and Methods}

\section{Animals}

All experiments were performed according to international guidelines and approved by the local experimental animal committee of the University Medical Center Utrecht (DEC-ABC, Utrecht). On postnatal day 9 (P9), C57Bl/6 mice pups were anaesthetized with isoflurane ( $4 \%$ induction, $2 \%$ maintenance), the right common carotid artery was occluded by thermo-cauterization, xylocaine $(100 \mathrm{mg} / \mathrm{ml}$; AstraZeneca, Zoetermeer, The Netherlands) was applied and the incision was closed. After a minimal $1 \mathrm{~h}$ recovery, the pups were exposed to $10 \% \mathrm{O}_{2}$ in $\mathrm{N}_{2}$ for $45 \mathrm{~min}$ and returned to their dams. Sham-control animals underwent anesthesia and incision only. LPS + HI or LPS+sham control animals received an intraperitoneal (i.p.) injection of LPS (List Biological Laboratories, Campbell, Calif., USA) at a dose of $0.5 \mathrm{mg} / \mathrm{kg} 14 \mathrm{~h}$ before surgery. Pups of both genders were used. Importantly, we did not observe any significant gender differences in any of the measured parameters. The experimental procedure resulted in a mortality rate of $8.4 \%$, there was no difference in the mortality rate between LPS+HI and HI animals. Mortality occurred during the experimental procedure and none of the animals died during follow-up.

For immunohistochemistry, animals were sacrificed by a pentobarbital overdose at $3 \mathrm{~h}$ or D1, 2, 3, 4, 5, 10 or 15 after the insult followed by transcardial perfusion with $4 \%$ paraformaldehyde in phosphate-buffered saline (PBS). Brains were postfixed and embedded in paraffin. For quantitative real-time reverse transcriptase PCR analysis, animals were sacrificed by decapitation directly before $\mathrm{HI}$ or at $3 \mathrm{~h}, \mathrm{D} 1,2$ or 3 postinsult. Contra- and ipsilateral brain hemispheres were collected and stored at $-80^{\circ} \mathrm{C}$.

\section{Histology}

Coronal paraffin brain sections $(8 \mu \mathrm{m})$ were cut at the level of the hippocampus and striatum (equivalent to -1.28 and $+0.38 \mathrm{~mm}$ from bregma in adult mice). Deparaffinized sections were stained with hematoxylin and eosin (HE; Klinipath, Duiven, The Netherlands) or were used for immunohistochemistry. In detail, deparaffinized sections that were used for microtubule-associated protein 2 (MAP2) or polymorphonuclear neutrophil (PMN) staining were boiled for $5 \mathrm{~min}$ in $10 \mathrm{mM}$ citrate buffer ( $\mathrm{pH} \mathrm{6.0)}$ ) for heat-induced antigen retrieval, before being blocked with $5 \%$ normal horse serum or $5 \%$ normal goat serum, respectively, followed by o/n incubation with mouse anti-MAP2 (1:1,000; Sigma-Aldrich, Steinheim, Germany) or rabbit anti-PMN (1:500; Accurate Antibodies, Westbury, N.Y., USA) antibodies. The next day, the sections were incubated with biotinylated horse anti-mouse or goat anti-rabbit (1:100; Vector Laboratories, Burlingame, Calif., USA) antibodies. Visualization was performed using Vectastain ABC kit (Vector 
Table 1. Primer sequences used for quantitative real-time reverse transcriptase PCR to measure cytokine/chemokine mRNA expression

\begin{tabular}{lll}
\hline Marker & Forward primer & Reverse primer \\
\hline GAPDH & TGAAGCAGGCATCTGAGGG & CGAAGGTGGAAGAGTGGGAG \\
$\beta-$ Actin & GATGCACAGTAGGTCTAAGTGGAG & CACTCAGGGCAGGTGAAACT \\
TNF- $\alpha$ & GCGGTGCCTATGTCTCAG & GCCATTTGGGAACTTCTCATC \\
IL- $\beta$ & CAACCAACAAGTGATATTCTCCATG & GATCCACACTCTCCAGCTGCA \\
IL-6 & TCTAATTCATATCTTCAACCAAGAGG & TGGTCCTTAGCCACTCCTTC \\
TGF- $\beta$ & GTGACAGCAAAGATAACAAAC & CTGAAGCAATAGTTGGTATCC \\
IL-10 & GCACCCACTTCCCAGTCG & GCATTAAGGAGTCGGTTAGCAG \\
MCP- 1 & GGTCCCTGTCATGCTTCTG & CATCTTGCTGGTGAATGAGTAG \\
CINC- 1 & AAAAGGTGTCCCCAAGTAACG & GTCAGAAGCCAGCGTTCAC
\end{tabular}

Laboratories) and diaminobenzamidine. We used PBS for all intermediary washing steps.

Subsequent paraffin sections were stained for mouse antiMBP. In short, deparaffinized sections were blocked with $20 \%$ normal horse serum in $\mathrm{PBS} / 0.5 \%$ Triton $\mathrm{X}-100$ before $\mathrm{o} / \mathrm{n}$ incubation with mouse-anti-MBP antibody (1:1,600, MBP; Sternberger Monoclonals Inc., Lutherville, Md., USA) followed by biotinylated horse anti-mouse (1:400; Vector Laboratories) antibody. Including an additional antigen retrieval step was not required for this staining. Visualization was performed using Vectastain ABC kit (Vector Laboratories) and diaminobenzamidine. We used PBS for all intermediary washing steps.

For HE, MAP2 and MBP staining, full-section images were captured with a Nikon D1 digital camera (Nikon, Tokyo, Japan). For HE and MAP2 staining the brain areas were outlined manually by an experienced scientist blinded to the experimental conditions, using image processing tools in Adobe Photoshop CS5 (Adobe Systems Inc., San Jose, Calif., USA). For quantification of MBP loss, image processing tools in ImageJ software (US National Institutes of Health, Bethesda, Md., USA; http://rsb.info.nih. gov/ij/) were used, in which the MBP-positive staining was converted to a binary signal and positive pixels were measured. Ipsilateral area loss was calculated as 1 - (area of ipsilateral staining/ area of contralateral staining) $\times 100 \%$. For analysis of corpus callosum thickness, photographs were taken of MBP-stained sections with a Zeiss Axio Lab A1 microscope and Icc5 camera, and analyzed using ZEN2012 software (Carl Zeiss, Oberkochen, Germany). MBP-positive fiber coherency was analyzed using the OrientationJ plugin for ImageJ (http://bigwww.epfl.ch/demo/orientation/). The fiber coherency was used as a measurement of the (dis) organization of MBP-positive fibers in the cortex. A higher fiber coherency indicates a more linear MBP-positive pattern resulting from a decrease in lateral arborization. PMN staining was quantified by counting the areal density of positive cells in the ipsilateral hemisphere by a scientist blinded to the experimental conditions.

For all immunofluorescent stainings, deparaffinized sections were boiled for $10-20 \mathrm{~min}$ in $10 \mathrm{mM}$ citrate buffer ( $\mathrm{pH}$ 6.0) for heat-induced antigen retrieval, before blocking them for $30 \mathrm{~min}$ with blocking buffer ( $0.1 \%$ saponin and $2 \%$ BSA in PBS), followed by $\mathrm{o} / \mathrm{n}$ incubation with rabbit anti-oligodendrocyte lineage transcription factor 2 (1:200, Olig2; Millipore, Billerica, Mass., USA), mouse anti-2' - $3^{\prime}$-cyclic nucleotide $3^{\prime}$-phosphodies- terase (1:200, CNPase; Abcam, Cambridge, UK) or rabbit antiionized calcium-binding adapter molecule 1 (1:200, Iba-1; Wako Chemicals, Richmond, Va., USA) followed by Alexa Fluor 488-conjugated secondary antibody (1:400; Molecular Probes, Eugene, Oreg., USA). PBS/0.5\% Tween was used as a washing buffer. Sections were counterstained with 4',6-diamidino-2-phenylindole (DAPI; Sigma-Aldrich) and embedded using Fluorsave (Calbiochem, Darmstadt, Germany). Fluorescent images were obtained using a Zeiss Axio Observer inverted microscope (Carl Zeiss) and were processed using ImageJ software. Images were analyzed by an observer blinded to the experimental conditions. For Olig2 and CNPase staining, 3 microscopic fields were analyzed per infarcted cortex and 5 microscopic fields per corpus callosum and cingulum area at the striatal level. The areal density of Olig2-positive cells was analyzed using the particle analysis nucleus counter plugin for ImageJ software. Data represent the mean value of areal density of Olig2-positive cells in 3-5 microscopic fields per square millimeter. For CNPase staining, the mean area of CNPase-positive pixels was measured per square millimeter using Image J software (threshold, histogram). For Iba-1 staining, 6 microscopic fields were analyzed per cortical and the hippocampal area and mean area of positive pixels were measured per square millimeter using ImageJ software (threshold, histogram). For CNPase or Iba-1, images of all animals were analyzed with similar threshold per staining.

For all immunohistochemical and immunofluorescent stainings, omitting the primary antibody resulted in negative staining of the brain sections, confirming the specificity of the staining. In addition, for all stainings used in this work, sections of all animals were always stained simultaneously to prevent variation in the procedure.

\section{Quantitative Real-Time Reverse Transcriptase PCR}

Total RNA was isolated from the brain samples using TRIzol ${ }^{\circledR}$ (Invitrogen, Paisley, UK) according to the manufacturer's protocol. cDNA was synthesized with SuperScript Reverse Transcriptase (Invitrogen). PCR was performed using the iQ5 Real-Time PCR Detection System (Bio-Rad Laboratories, Veenendaal, The Netherlands) for tumor necrosis factor- $\alpha$ (TNF- $\alpha$ ), interleukin (IL)-1 $\beta$, IL-6, transforming growth factor- $\beta$ (TGF- $\beta$ ), IL-10, monocyte chemoattractant protein 1 (MCP-1) and cytokine-induced neutrophil chemoattractant 1 (CINC-1; for primer sequences, see table 1). To confirm appropriate amplification, the
80
Dev Neurosci 2015;37:78-94 DOI: $10.1159 / 000368770$
Bonestroo/Heijnen/Groenendaal/van Bel/ Nijboer 
size of PCR products was verified on gel. Data were individually normalized for the mean of the relative expression of glyceraldehyde 3-phosphate dehydrogenase (GAPDH) and $\beta$-actin.

\section{Statistical Analysis}

All analyses were performed in a blinded setup. Statistical analyses were performed using SPSS (version 20.0) and GraphPad Prism 5 (GraphPad Software, La Jolla, Calif., USA) software. All data are expressed as mean \pm SEM and were analyzed by one- or two-way ANOVA with Bonferroni post hoc tests. $\mathrm{p}<0.05$ was considered statistically significant.

\section{Results}

\section{Cerebral Gray Matter Injury}

$\mathrm{HI}$ brain damage was induced in $\mathrm{P} 9$ mouse pups by unilateral occlusion of the right carotid artery and $45 \mathrm{~min}$ of systemic hypoxia (designated as the 'HI' group). To investigate the additional effect of a systemic inflammation on HI-induced brain damage, animals in the LPS+HI group received an i.p. injection of LPS at a dose of $0.5 \mathrm{mg} /$ $\mathrm{kg} 14 \mathrm{~h}$ before the HI insult. Mice were terminated at several time points after HI, from $3 \mathrm{~h}$ until D15, to observe the development of brain injury over time after HI or LPS+HI (fig. 1).

First we determined the effect of HI and LPS+HI on cerebral volume loss and possible cyst formation by using HE staining. During the first 2 days after the induction of $\mathrm{HI}$, no clear volume loss of the ipsilateral hemisphere was observed in the HI or LPS+HI groups (fig. 1a, c). Starting at D3, HI alone caused a small reduction in ipsilateral cortical volume with disintegration of the hippocampal architecture without formation of a cyst (i.e. complete loss of tissue/an acellular lesion; fig. 1c). No significant increase in ipsilateral volume loss was observed in $\mathrm{HI}$ animals between D3 and D15 and, consistent with this finding, none of the HI-mice displayed cyst formation (fig. 1a, c). When HI was preceded by LPS, however, a significant increase in ipsilateral volume loss was observed com-

Fig. 1. Development of HI-induced cerebral volume loss and neuronal damage at different time points after HI or LPS+HI. Mouse pups were subjected to HI at P9 (HI). Sham-operated littermates (Sham) were used as controls. Animals received an i.p. LPS injection $14 \mathrm{~h}$ before the HI insult or sham operation (LPS+HI or LPS, respectively). a Cerebral tissue loss was measured by analyzing loss of $\mathrm{HE}$ staining in the ipsilateral hemisphere from $3 \mathrm{~h}$ up to D15 postinsult. Two-way ANOVA: $\mathrm{F}_{(2,114)}=182.1, \mathrm{p}<0.001$; time effect $\mathrm{F}_{(7,114)}=$ 39.1, $\mathrm{p}<0.001$; interaction $\mathrm{F}_{(14,114)}=15.9, \mathrm{p}<0.001$. b Neuronal damage in the ipsilateral hemisphere was assessed by measuring loss of MAP2 staining at the hippocampal level at the indicated time

Brain Injury after Inflammatory Perinatal Asphyxia pared to $\mathrm{HI}$ littermates starting at D3 postinsult (32.4 \pm $3.8 \%$ ipsilateral volume loss in LPS+HI mice vs. $17.0 \pm$ $2.4 \%$ in $\mathrm{HI}$ animals, $\mathrm{p}<0.01$; fig. 1a, c). After LPS $+\mathrm{HI}$, macroscopically visible cystic lesions developed from D4 onwards, located in the hippocampal and cortical area. Moreover, the amount of ipsilateral volume loss significantly increased over time in LPS+HI versus HI mice $(42.5 \pm 5.0$ vs. $15.5 \pm 1.0 \%$ at $\mathrm{D} 4,46.6 \pm 3.3$ vs. $16.3 \pm 3.9 \%$ at $\mathrm{D} 5,65.2 \pm 3.7$ vs. $30.8 \pm 6.1 \%$ at $\mathrm{D} 10$ up to $55.7 \pm 4.8$ vs. $24.5 \pm 8.7 \%$ at $\mathrm{D} 15, \mathrm{p}<0.001$ for all time points; fig. $1 \mathrm{a}$, c). Importantly, no changes in volume loss were observed in the contralateral hemisphere of HI or LPS+HI animals compared to sham-control littermates.

MAP2 staining was performed to determine the specific development of gray matter injury after $\mathrm{HI}$ and LPS+HI. At $3 \mathrm{~h}$ postinsult, HI resulted in ipsilateral MAP2 loss of $23.1 \pm 4.7 \%$, which was limited to the hippocampal area (fig. 1b, c). Interestingly, no further loss of MAP2 staining was detectable from $3 \mathrm{~h}$ until D15 after HI (fig. 1b, c). LPS injection before HI resulted in a significant increase in MAP2 loss, which was located in the ipsilateral hippocampus, cortex and striatum (fig. 1b, c). Enhanced MAP2 loss after LPS+HI was already present as early as D1 postinsult (LPS+HI vs. HI, $44.7 \pm 7.9$ vs. $21.3 \pm 5.0 \%$, p < 0.01 ) and showed a gradual increase over time up to $63.3 \pm$ 6.4 vs. $24.0 \pm 4.8 \%$ MAP2 loss at D15 in LPS+HI vs. HI animals $(\mathrm{p}<0.001)$. Anesthesia and incision only did not result in neuronal damage since we did not observe any MAP2 loss in sham-control animals at any of the measured time points. LPS injection alone without HI did not induce any MAP2 or volume loss at 6 days (P14) after injection compared to sham-operated littermates (fig. 1d).

\section{Cerebral White Matter Injury}

Myelination of the brain is hardly detectable in the first postnatal week of rodent brain development. We first determined at which postnatal day MBP expression was high enough to reliably study the effects of HI and LPS+HI

points postinsult. Two-way ANOVA: $\mathrm{F}_{(2,120)}=160.5, \mathrm{p}<0.001$. There was no significant time effect or interaction. c Representative examples of HE and MAP2 staining at the hippocampal level in brains of sham-operated, $\mathrm{HI}$ and LPS $+\mathrm{HI}$ animals at the indicated time points after insult. d Representative examples of HE and MAP2 staining at the hippocampal level in brains of sham-operated and LPS-alone animals at 6 days after injection, i.e. P14. Sham $\mathrm{n}=5-8$, HI $n=6-8$, LPS + HI $n=6-7$ per time point. Data are expressed as mean \pm SEM. Data were analyzed using two-way ANOVA with Bonferroni post hoc tests. LPS+HI vs. HI: ${ }^{* *} \mathrm{p}<0.01{ }^{* * *} \mathrm{p}<0.001$; HI vs. sham: ${ }^{\text {}} \mathrm{p}<0.05,{ }^{\# \#} \mathrm{p}<0.01,{ }^{\# \# \#} \mathrm{p}<0.001$.

(For figure see next page.) 


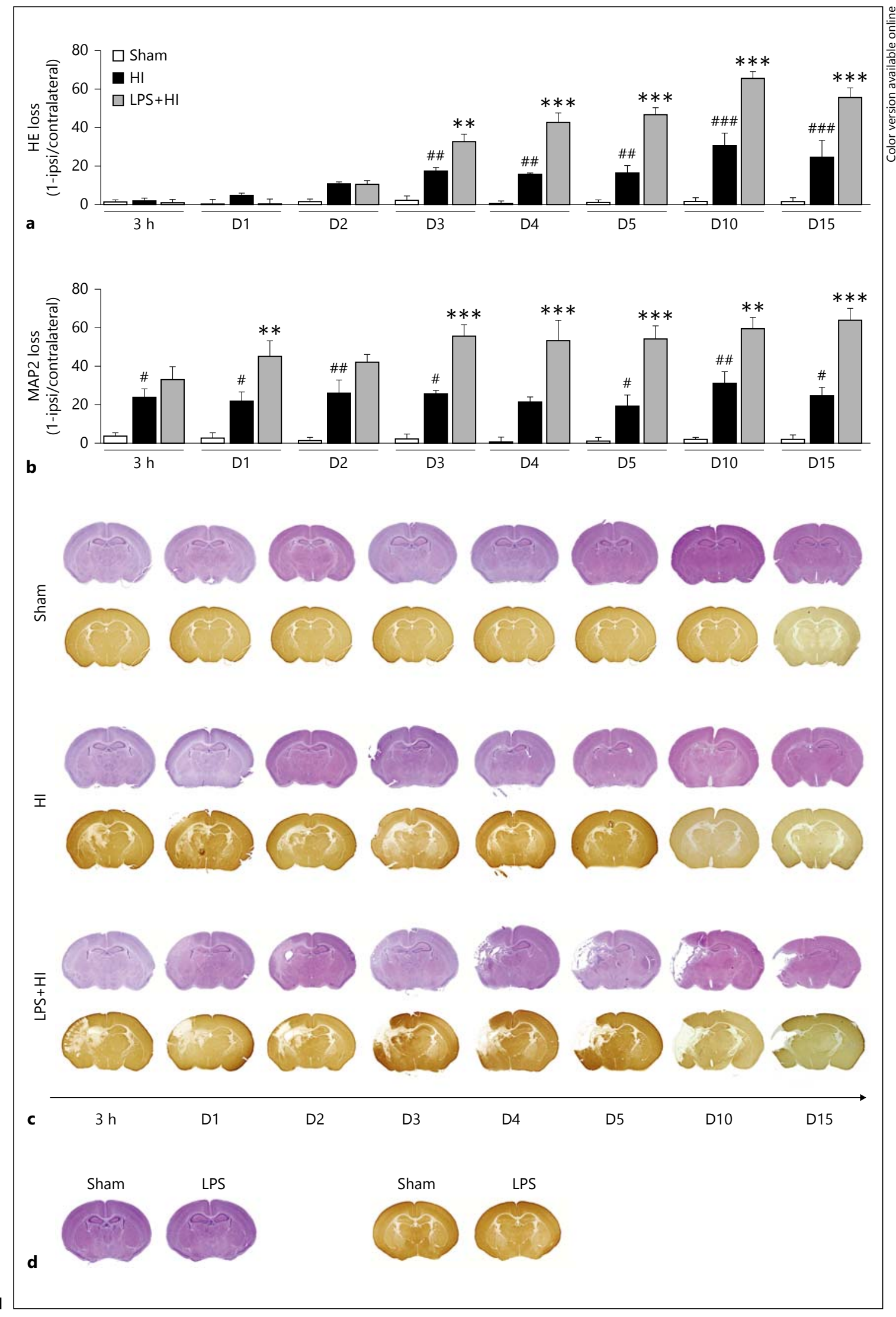


Fig. 2. Development of HI-induced cerebral white matter injury at different time points after HI or LPS+HI. a MBP expression in both hemispheres in sham-operated control animals (Sham) from P9 up to P24 (D15 after insult). b Mouse pups were subjected to HI at P9 (HI). Sham-operated littermates were used as controls. Animals received an i.p. LPS injection $14 \mathrm{~h}$ before the HI insult or sham operation (LPS+HI or LPS, respectively). Cerebral white matter damage was measured by analyzing the loss of MBP staining at hippocampal level in the ipsilateral hemisphere from D3 up to D15 postinsult. Two-way ANOVA: $\mathrm{F}_{(2,72)}=115.2, \mathrm{p}<0.001$. There was no significant time effect or interaction. c Representative examples of MBP staining at hippocampal level in brains of sham-operated, $\mathrm{HI}$ and LPS+HI animals at the indicated time points after insult. d Representative examples of MBP staining at hippocampal level in brains of sham-operated and LPSalone animals at 6 days after injection, i.e. P14. Sham $\mathrm{n}=5-8, \mathrm{HI} \mathrm{n}=6-8$, LPS $+\mathrm{HI} \mathrm{n}=$ 6-7 per time point. Data are expressed as mean \pm SEM. Data were analyzed using two-way ANOVA with Bonferroni post hoc tests. LPS+HI versus HI: ${ }^{*} \mathrm{p}<0.05$, ${ }^{* *} \mathrm{p}<0.01,{ }^{* * *} \mathrm{p}<0.001 ;$ HI vs. sham ${ }^{\#} \mathrm{p}<$ $0.05,{ }^{\# \#} \mathrm{p}<0.01$, \#\# $\mathrm{p}<0.001$. A.U. $=$ Arbitrary unit.

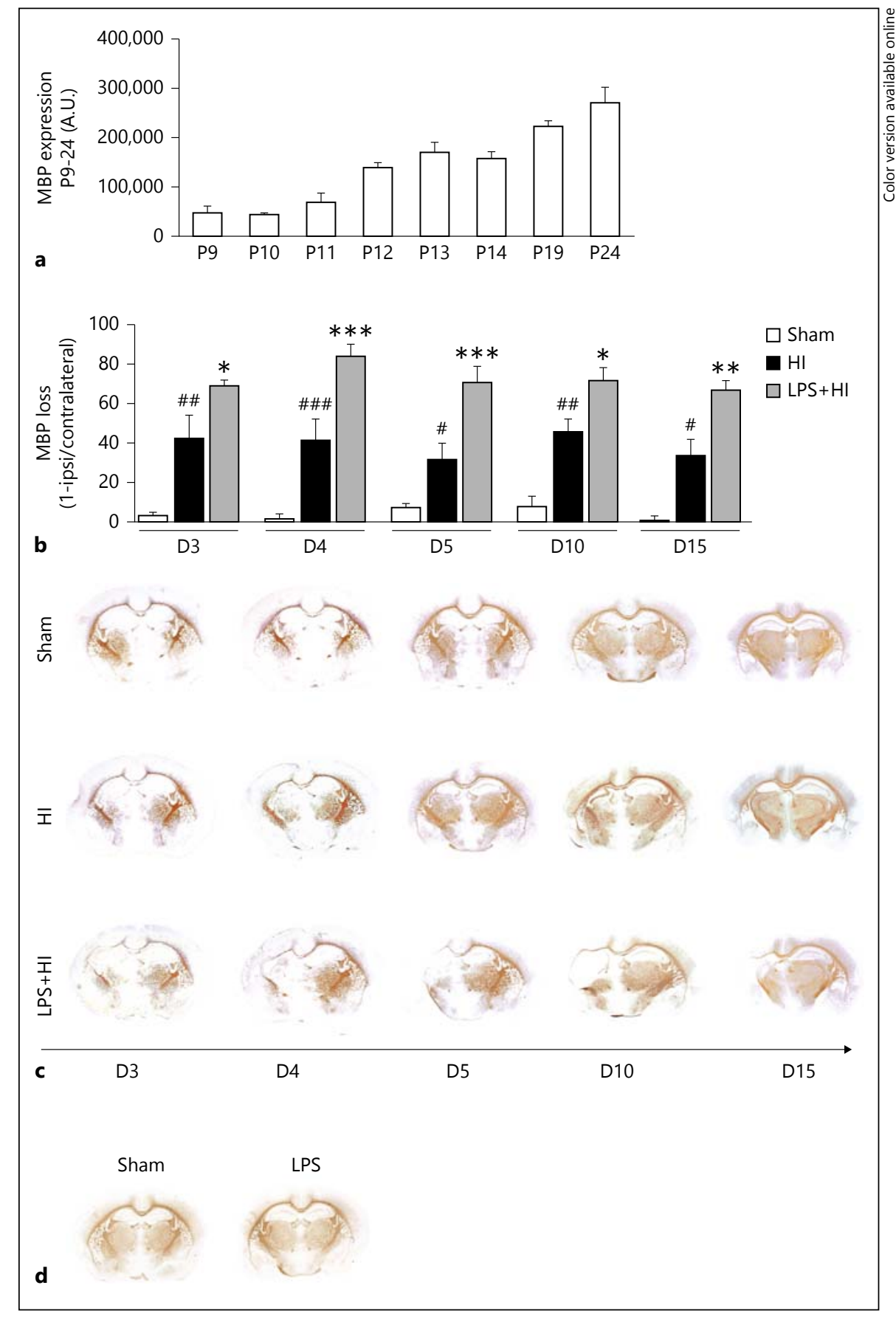

on MBP loss as a measure of white matter damage (fig. 2a). To this end, we quantitatively measured MBP expression over time in both hemispheres of sham-control animals. Figure 2a shows that MBP expression in the mouse brain was very low until P11. From P12-24, MBP expression gradually increased (fig. 2a). The effects of $\mathrm{HI}$ and LPS+HI on MBP loss were therefore measured from D3 (P12) up to D15 (P24) postinsult. HI resulted in stable white matter damage from D3 to D15, i.e. we measured $41.9 \pm 12.2 \%$ ipsilateral MBP loss at D3 without any further increase up to D15 (fig. 2b, c). After combined exposure to LPS+HI, ipsilateral MBP loss was significantly increased: $69.0 \pm$ $2.7 \%$ following LPS+HI vs. $41.9 \pm 12.2 \%$ after $\mathrm{HI}$ at D3 postinsult ( $\mathrm{p}<0.05$; fig. $2 \mathrm{~b}, \mathrm{c})$. Interestingly, in the LPS $+\mathrm{HI}$ 
group the loss of MBP staining remained constant over the period of D3-15 (fig. 2b, c). No MBP loss was observed in sham-control littermates or in the contralateral hemisphere of HI or LPS+HI animals. LPS injection alone without $\mathrm{HI}$ did not induce any MBP loss at 6 days (P14) after injection compared to sham-operated littermates (fig. 2d).

Next, we studied the effects of HI and LPS+HI on white matter integrity. It is known that $\mathrm{HI}$ can induce changes in corpus callosum volume, which is associated with lateralizing motor deficits $[22,23]$. Therefore, we measured the thickness of the corpus callosum after HI and LPS+HI at the striatal level, both at the midline and in the contra- and ipsilateral hemisphere at D10 postinsult. $\mathrm{HI}$ induced a nonsignificant thinning of the corpus callosum compared to sham-operated littermates at the midline $(83.1 \pm 10.4$ vs. $54.6 \pm 14.4 \mu \mathrm{m}$, HI vs. sham; fig. 3a, c). Administration of LPS prior to HI caused a significant reduction in the thickness of the corpus callosum at the midline compared to sham-control levels (83.1 \pm 10.4 vs. $39.9 \pm 6.2 \mu \mathrm{m}, \mathrm{p}<0.05$; fig. $3 \mathrm{a}, \mathrm{c})$. Additionally, in LPS+HI animals loss of MBP staining within the corpus callosum was clearly extending towards the ipsilateral hemisphere, in particular in the ventral layer of the corpus callosum. The thickness of the corpus callosum in the ipsilateral hemisphere was $23.1 \pm 2.6$ vs. $46.7 \pm 9.5 \mu \mathrm{m}$ (LPS+HI vs. sham, $\mathrm{p}<0.05$; fig. $3 \mathrm{a}, \mathrm{c}$ ). HI alone did not induce significant thinning of the corpus callosum in the ipsilateral hemisphere (fig. 3a, c). No significant differences in corpus callosum thickness were measured in the contralateral hemisphere of sham-operated, HI or LPS+HI animals.

We have shown before that $\mathrm{HI}$ can induce a decline in lateral arborization of MBP-positive fibers in the ipsilateral cingulum, defined as an increase in fiber coherency demonstrated by a more linear MBP pattern [23]. Figure $3 \mathrm{~d}$ and e show that HI did not result in a significant increase in MBP-positive fiber coherency (indicating no significant reduction in lateral arborization of MBP-positive fibers) within the cingulum at D10 postinsult compared to sham-operated littermates. LPS administration before HI, however, caused a significant decrease in lateral arborization of MBP-positive fibers, resulting in strongly increased MBP-positive fiber coherency compared to $\mathrm{HI}$ and sham at D10 $(0.15 \pm 0.02$ vs. $0.07 \pm 0.02$ or $0.05 \pm 0.01 \%$, LPS + HI vs. HI or sham, $\mathrm{p}<0.05$ or $\mathrm{p}<$ 0.01 ; fig. $3 \mathrm{~d}, \mathrm{e})$.

Next, we examined whether LPS affects oligodendrocyte numbers after HI. We stained the brains of shamoperated, HI and LPS+HI mice with an antibody directed against Olig2, which is expressed throughout the oligodendrocyte lineage and thus identifies the entire pool of oligodendrocytes, and with an antibody directed against CNPase that identifies more mature (myelinating) oligodendrocytes. HI significantly increased the areal density of Olig2-positive cells in the ipsilateral infarct area compared to sham-operated animals at D10 (152.0 $\pm 17.1 \mathrm{vs.}$ $79.0 \pm 2.4$ cells $/ \mathrm{mm}^{2}, \mathrm{p}<0.001$; fig. $\left.4 \mathrm{a}, \mathrm{c}\right)$. HI did not induce changes in the areal density of Olig2-positive cells in the corpus callosum/cingulum area (fig. 4b). After LPS+HI, a significantly higher areal density of Olig2-positive cells was observed in the infarct area compared to HI littermates $\left(221.5 \pm 24.1\right.$ vs. $152.0 \pm 17.1$ cells $/ \mathrm{mm}^{2}, \mathrm{p}<$ 0.01 ; fig. $4 \mathrm{a}, \mathrm{c}$ ), whereas LPS $+\mathrm{HI}$ also did not induce changes in the areal density of Olig2-positive cells in the corpus callosum/cingulum area (fig. $4 \mathrm{~b}$ ). Figure $4 \mathrm{~d}-\mathrm{f}$ show that HI caused a significant decrease in more mature myelinating oligodendrocytes measured by a decrease in CNPase-positive signal in the ipsilateral infarct area $(p<0.001)$ and a small, nonsignificant decrease of CNPase signal in the corpus callosum/cingulum area compared to sham-control animals. LPS administration before $\mathrm{HI}$, however, resulted in a dramatic decrease of CNPase-positive signal in the infarct area $(\mathrm{p}<0.001)$ and corpus callosum/cingulum area $(\mathrm{p}<0.01)$ compared to HI (fig. 4d-f).

\section{Cerebral Inflammatory Response}

To investigate the effect of LPS on the HI-induced cerebral inflammatory response, we analyzed pro- and antiinflammatory cytokine and chemokine mRNA expression in the brain at $3 \mathrm{~h}, \mathrm{D} 1 \mathrm{(}(24 \mathrm{~h}), 2$ and 3 postinsult. Figure $5 \mathrm{a}$ shows that $\mathrm{HI}$ induced an ipsilateral upregulation of TNF- $\alpha$ mRNA expression compared to sham-control levels within $3 \mathrm{~h}$ up to D1 post-HI. The expression of IL-1 $\beta$ and IL-6 mRNA showed a modest, nonsignificant increase compared to sham-control levels at $3 \mathrm{~h}$ and $\mathrm{D} 1$ postinsult. At D2 and D3 after HI, TNF- $\alpha$, IL- $1 \beta$ and IL- 6 mRNA expression levels declined to sham-control levels (fig. 5a). When HI was preceded by LPS injection, however, TNF- $\alpha$ mRNA expression was strongly increased compared to HI. In more detail, at $3 \mathrm{~h}$ postinsult, TNF- $\alpha$ expression increased 34- vs. 16-fold in the LPS+HI vs. HI animals compared to sham levels $(\mathrm{p}<0.001)$. Moreover, TNF- $\alpha$ mRNA expression remained significantly elevated until D3 in LPS+HI mouse pups (fig. 5a). In addition, LPS+HI induced a significant upregulation of IL- $1 \beta$ mRNA expression at $3 \mathrm{~h}$ and $\mathrm{D} 1$ compared to $\mathrm{HI}(\mathrm{p}<0.01$ and $\mathrm{p}<0.001)$, which returned to baseline at D2 postinsult. LPS administration before HI significantly enhanced IL-6 expression 
Fig. 3. Effects of HI and LPS+HI on thinning of the corpus callosum (CC) and arborization of MBP-positive fibers in the cingulum. Myelination in the CC and cingulum was measured at the striatal level by analyzing staining for MBP at 10 days postinsult. a CC thickness in micrometers measured at the midline and in the ipsilateral part of the CC in sham-operated, HI and LPS+HI mouse pups. One-way ANOVA: $\mathrm{F}_{(2,14)}=3.8, \mathrm{p}<0.05$, and $\mathrm{F}_{(2,13)}=2.8, \mathrm{p}<$ 0.05 , for the midline and ipsilateral measurements, respectively. b Schematic coronal brain picture illustrating where photographs were taken in the CC (middle lower square) and cingulum (left upper square). c Representative photographs of the MBPstained CC in the different experimental groups. $\mathbf{d} \mathrm{MBP}$-positive fiber coherency in the ipsilateral cingulum, as a measure of (dis)organization of MBP-positive fibers in sham-operated, HI and LPS+HI mouse pups. Data represent the mean value of MBP-positive fiber coherency in the cingulum measured in 4 areas of one microscopic field. One-way ANOVA: $\mathrm{F}_{(2,13)}=8.0, \mathrm{p}<$ 0.01. e Representative pictures of MBP staining in the ipsilateral cingulum of sham-operated, HI and LPS+HI animals. Sham $n=5$, HI $n=6$, LPS + HI $n=6$. Data are expressed as mean \pm SEM. Data were analyzed using one-way ANOVA with Bonferroni post hoc tests. ${ }^{*} \mathrm{p}<0.05,{ }^{* *} \mathrm{p}<$ 0.01 .

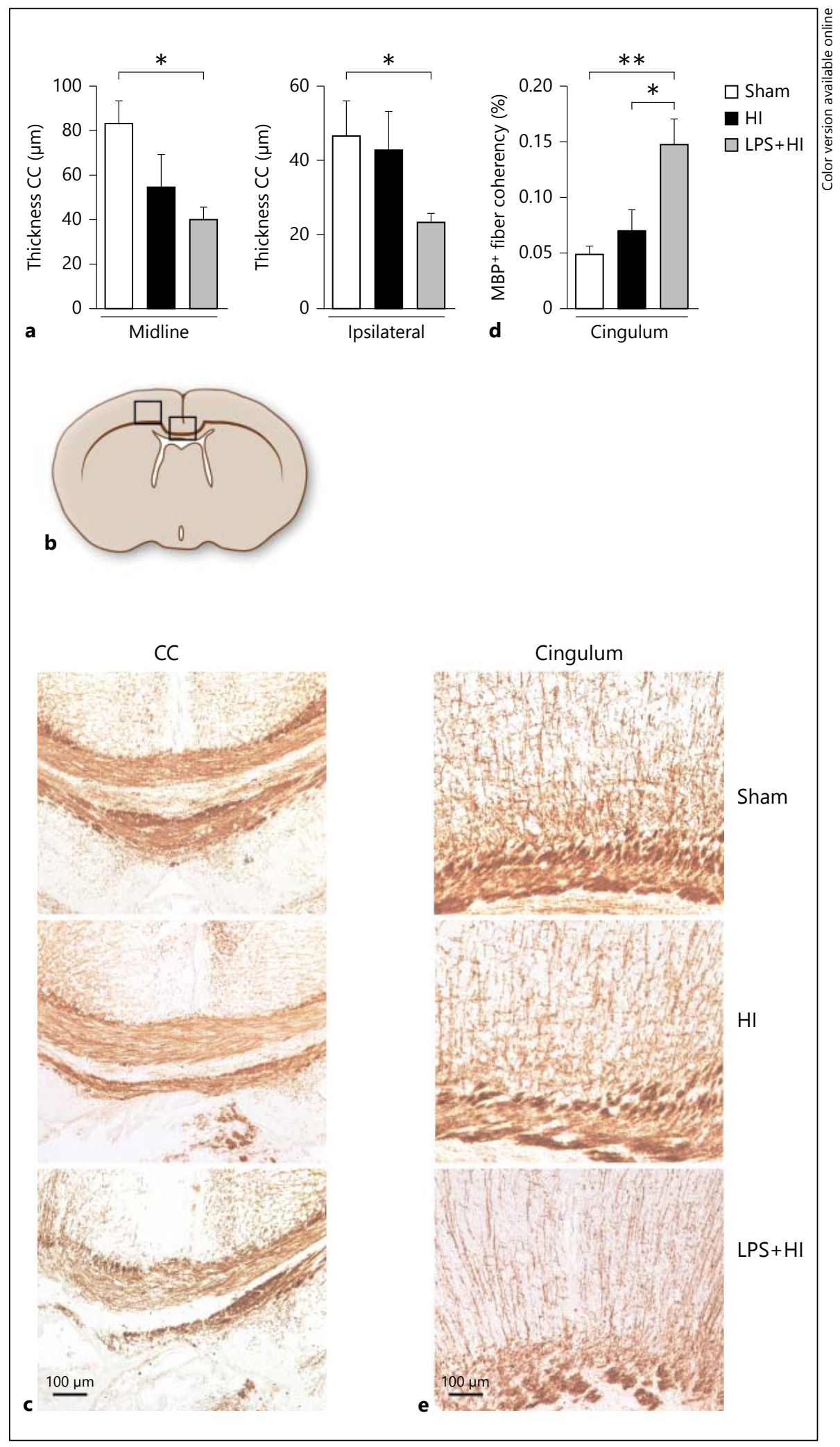


Fig. 4. HI- and LPS+HI-induced changes in the number of oligodendrocytes at 10 days postinsult. The areal density of oligodendrocytes throughout the lineage was measured by analyzing the expression of Olig2 staining. CNPase was used as a marker for more mature myelinating oligodendrocytes. a Areal density of Olig2-positive cells in the infarcted cortex at the striatal level of sham-operated, HI and LPS+HI mouse pups. Data represent the mean value of the areal density of Olig2-positive cells in 3 microscopic fields. Two-way ANOVA: $\mathrm{F}_{(2,28)}=11.9, \mathrm{p}<0.001$. b Areal density of Olig2-positive cells in the corpus callosum (CC) and cingulum at the striatal level of sham-operated, HI and LPS+HI mouse pups. Data represent the mean value of the areal density of Olig2-positive cells in 5 microscopic fields. Two-way ANOVA: $\mathrm{F}_{(2,28)}=1.2, \mathrm{p}=0.332$. c Representative examples of Olig2-positive cells in the infarcted cortex in brains of sham-operated, $\mathrm{HI}$ and LPS+HI animals. d CNPase-positive signal in the infarcted cortex at the striatal level of sham-operated, HI and LPS+HI mouse pups. Data represent the mean value of the area of positive signal in 3 microscopic fields. Two-way analysis of variance $\mathrm{F}_{(2,28)}=23.5, \mathrm{p}<0.001$. e CNPase-positive signal in the $\mathrm{CC}$ and cingulum at the striatal level of sham-operated, HI and LPS+HI mouse pups. Data represent the mean value of the area of positive signal in 5 microscopic fields. Two-way analysis of variance $\mathrm{F}_{(2,28)}=10.7, \mathrm{p}<0.001$. f Representative images of CNPase staining in the infarcted cortex in brains of sham-operated, $\mathrm{HI}$ and LPS $+\mathrm{HI}$ animals. Sham $\mathrm{n}=5$, HI $\mathrm{n}=6$, LPS $+\mathrm{HI} \mathrm{n}=6$. Data are expressed as mean \pm SEM. Data were analyzed using two-way ANOVA with Bonferroni post hoc tests. ${ }^{* *} \mathrm{p}<0.01,{ }^{* * *} \mathrm{p}<0.001$. n.s. $=$ Nonsignificant; $\mathrm{c}=$ contralateral hemisphere; $\mathrm{i}$ = ipsilateral hemisphere; pos = positive.

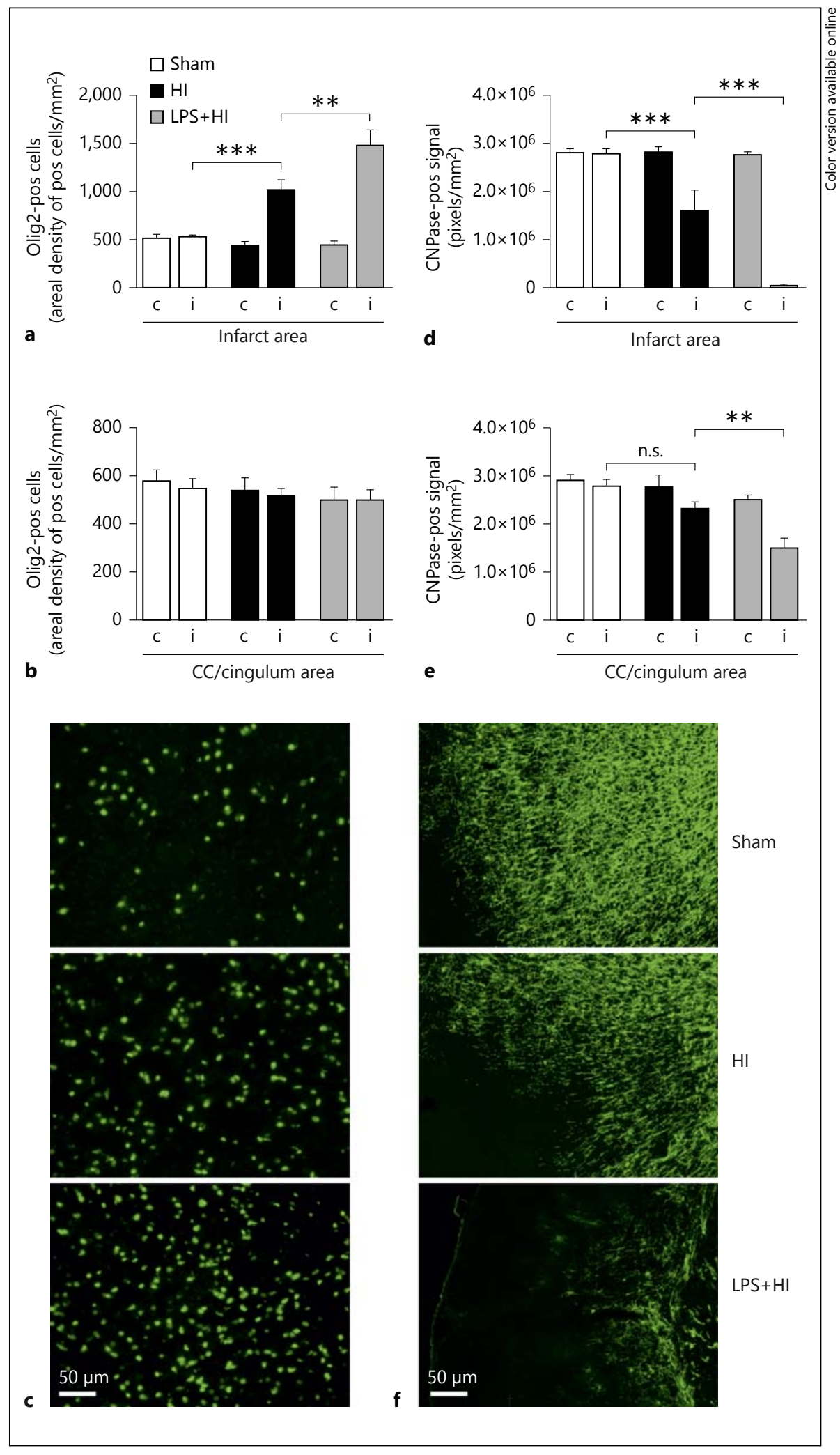




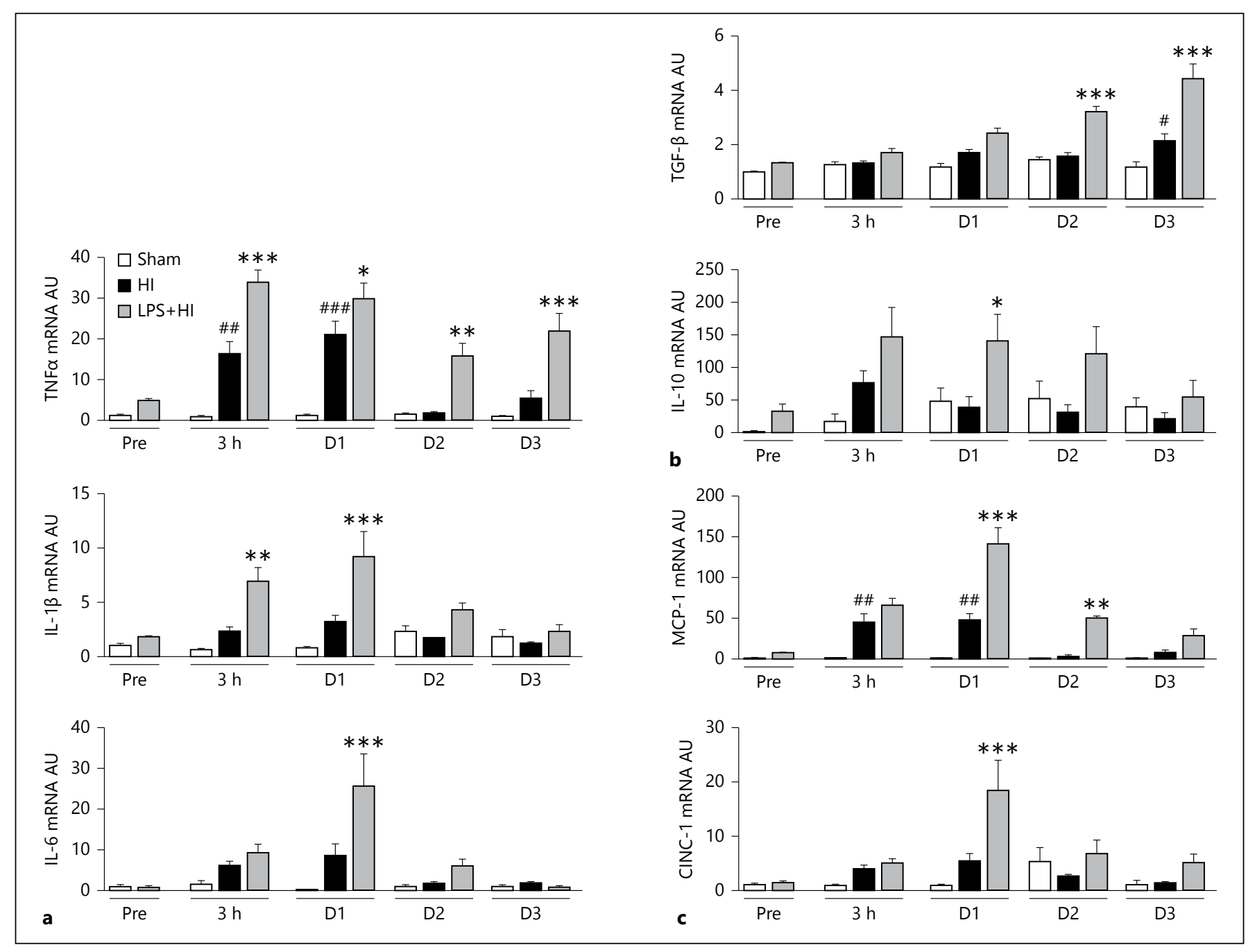

Fig. 5. Ipsilateral mRNA expression of pro- and anti-inflammatory cytokines and chemokines at different time points postinsult. Cerebral mRNA expression of cytokines and chemokines was measured by quantitative real-time reverse transcriptase PCR at the indicated time points. a Ipsilateral mRNA expression of the proinflammatory cytokines TNF- $\alpha$, IL- $1 \beta$ and IL-6. Two-way ANOVA: TNF- $\alpha \mathrm{F}_{(2,63)}=63.6, \mathrm{p}<0.001$; IL- $1 \beta \mathrm{F}_{(2,57)}=19.4, \mathrm{p}<$ 0.001 ; IL- $6 \mathrm{~F}_{(2,59)}=11.9, \mathrm{p}<0.001$. b Ipsilateral mRNA expression of the anti-inflammatory factors TGF- $\beta$ and IL-10. Two-way ANOVA: TGF- $\beta \mathrm{F}_{(2,63)}=55.0, \mathrm{p}<0.001$; IL-10 $\mathrm{F}_{(2,63)}=9.5, \mathrm{p}<$ 0.001 . c Ipsilateral mRNA expression of the chemokines MCP-1 and CINC-1. Two-way ANOVA: MCP-1 $\mathrm{F}_{(2,58)}=58.0, \mathrm{p}<0.001$;

at D1 (26- vs. 9-fold, LPS+HI vs. HI compared to sham levels, $\mathrm{p}<0.05)$. IL-6 mRNA levels sharply declined at D2 in the HI and LPS+HI group (fig. 5a). Thus, our data show that LPS+HI resulted in a significantly increased and prolonged upregulation of proinflammatory cytokines when compared to HI only (fig. 5a).

CINC- $1 \mathrm{~F}_{(2,62)}=10.5, \mathrm{p}<0.001$. Data were individually normalized for the mean of the relative expression of GAPDH and $\beta$-actin. Expression levels are presented relative to levels in sham-operated animals at the time point 'Pre', which were set at 1 . No significant changes in mRNA expression were observed in the contralateral hemispheres of HI or LPS $+\mathrm{HI}$ animals compared to levels in shamoperated littermates. Sham $n=5, \mathrm{HI} n=6-8$, LPS $+\mathrm{HI} n=6-8$ per time point. Data are expressed as mean \pm SEM. Data were analyzed using two-way ANOVA with Bonferroni post hoc tests. LPS+HI vs. HI: ${ }^{*} \mathrm{p}<0.05,{ }^{* *} \mathrm{p}<0.01,{ }^{* * *} \mathrm{p}<0.001$; HI vs. sham ${ }^{\#} \mathrm{p}<0.05$, \#\# $\mathrm{p}<0.01$, \#\#\# $\mathrm{p}<0.001$. Pre $=$ Animals terminated before hypoxia; $\mathrm{AU}=$ arbitrary units.

Next we studied the expression of the anti-inflammatory mediators TGF- $\beta$ and IL- 10 after HI and LPS+HI. HI induced a moderate upregulation of the anti-inflammatory gene TGF- $\beta$ at D3 postinsult in the ipsilateral hemisphere ( $p<0.05$; fig. $5 b)$. LPS administration prior to HI resulted in a significant ipsilateral upregulation of TGF- $\beta$ 
at $\mathrm{D} 2$ and $\mathrm{D} 3$ postinsult compared to $\mathrm{HI}$ ( $\mathrm{p}<0.0001$ and $\mathrm{p}<0.01$; fig. 5b). For IL-10 mRNA expression, we observed that $\mathrm{HI}$ did not induce a significant upregulation compared to sham-control levels. LPS+HI induced a strong upregulation of IL-10 expression at $3 \mathrm{~h}, \mathrm{D} 1$ and D2 which declined to baseline at $\mathrm{D} 3$ post-insult. HI-induced IL-10 expression was significantly higher in LPS+HI brains compared to $\mathrm{HI}$ at $\mathrm{D} 1$ postinsult ( $\mathrm{p}<0.05$; fig. $5 \mathrm{~b}$ ).

Additionally, we determined the effect of $\mathrm{HI}$ and LPS+HI on the expression of MCP-1 and CINC-1, two important chemoattractants for influx of macrophages and neutrophils, respectively. After HI, we observed a 45and 47 -fold increase in cerebral MCP-1 expression at $3 \mathrm{~h}$ and D1 postinsult compared to sham-control levels (fig. 5c). The expression of CINC-1 showed a small, nonsignificant increase at $3 \mathrm{~h}$ and D1 (4- vs. 6-fold, HI compared to sham levels). When HI was preceded by an LPS injection, increased expression of MCP-1 was observed at D1 up to D2 postinsult ( $\mathrm{p}<0.001$ and $\mathrm{p}<0.01$ ), with a peak at D1 (fig. 5c). At D1, LPS+HI induced significantly higher levels of CINC-1 ( $\mathrm{p}<0.001)$ compared to HI.

The data presented in figure 5 show that when $\mathrm{HI}$ is preceded by LPS both the proinflammatory as well as anti-inflammatory cytokine/chemokine response in the brain is increased and prolonged. Notably, LPS injection without $\mathrm{HI}$ resulted in a small but nonsignificant upregulation of TNF- $\alpha$, IL-1 $\beta$, IL-10 and MCP-1 when measured $14 \mathrm{~h}$ after the injection ('Pre' groups; fig. 5a-c).

To determine the cerebral activation/influx of microglia/macrophages and neutrophils, we performed Iba-1 and PMN stainings, respectively, on brain sections of sham-operated, LPS alone, HI and LPS+HI mouse pups. Figure $6 \mathrm{a}$ and $\mathrm{c}$ show that after HI Iba-1-positive cells displayed a more activated phenotype, but Iba-1-positive signal was not significantly increased. LPS $+\mathrm{HI}$ resulted in a significant increase in Iba-1-positive signal with a concomitant increased intensity of Iba-1 staining per cell, indicating an increased activation state of macrophages/ microglia in the ipsilateral cortical and hippocampal area at $\mathrm{D} 2$ postinsult $(\mathrm{p}<0.05$; fig. 6a) [24-26]. The Iba-1-positive signal declined at D15 postinsult (fig. 6a). Figure 6c illustrates that Iba-1-positive cells in sham-operated mice displayed a resting state phenotype with ramified morphology, whereas the Iba-1-positive cells in HI and LPS+HI animals showed a more activated state with amoeboid shape and retracted processes. Importantly, LPS injection alone without $\mathrm{HI}$ did not induce an increase in Iba-1-positive signal at D2 (3 days after injection) and Iba-1-positive cells displayed a resting state phenotype like sham-operated littermates (fig. 6c).
To assess neutrophil influx into the brain parenchyma, a PMN staining was performed during the first 3 days following the insult. Neutrophils were not detected in the brains of sham-control animals (fig. 6d, e). Following HI, a small increase in the areal density of neutrophils was observed in the right cerebral hemisphere at D1-3. LPS+HI resulted in a massive influx of neutrophils, peaking at D1, which was significantly increased compared to HI only $(\mathrm{p}<$ 0.001 ). Neutrophil areal density declined but remained slightly elevated until D3 (fig. 6d). Neutrophils were specifically observed in the damaged cortex, with minimal numbers in the hippocampal area. A large number of infiltrating neutrophils were observed within the brain parenchyma (outside blood vessels), indicating the diapedesis of these cells. LPS injection alone without HI did not induce influx of neutrophils into the brain at D1 (2 days after injection) compared to sham-operated littermates (fig. 6e).

\section{Discussion}

Over recent years, the role of inflammation in the development of neonatal brain damage has become a matter of great interest. Antenatal infections are recognized as an important risk factor for adverse outcome in term and preterm neonates [27-35]. The combined exposure to perinatal inflammation and neonatal $\mathrm{HI}$ results in a complex interplay of various signaling pathways of which the exact underlying mechanism has not been elucidated yet. Experimental peripheral inflammation induced by LPS is known to induce an inflammatory cerebral state leading to sensitization of the brain to HI injury $[15,16,19,21$, 36, 37]. Possible mechanisms via which peripheral LPS can sensitize the brain have been described extensively and consist of: (i) stimulation of endothelial cells of the blood-brain barrier (BBB), (ii) activation/priming of microglia via immune signals produced by endothelial and choroid plexus cells at the level of the BBB, (iii) direct transport of peripheral inflammatory molecules across the $\mathrm{BBB}$, (iv) increased permeability of the BBB, which enhances the influx of peripheral immune cells into the brain, (v) stimulation of the vagal nerve by peripheral cytokines leading to signaling in the brain and (vi) the effects of inflammation on G-protein-coupled receptor signaling in the brain $[17,38-41]$. In the present study, we carefully characterized the sensitizing effect of LPS on the severity, appearance and especially timing of HI-induced brain injury.

We have described here that an inflammatory state before $\mathrm{HI}$ not only aggravates neuronal damage measured
Bonestroo/Heijnen/Groenendaal/van Bel/ Nijboer 


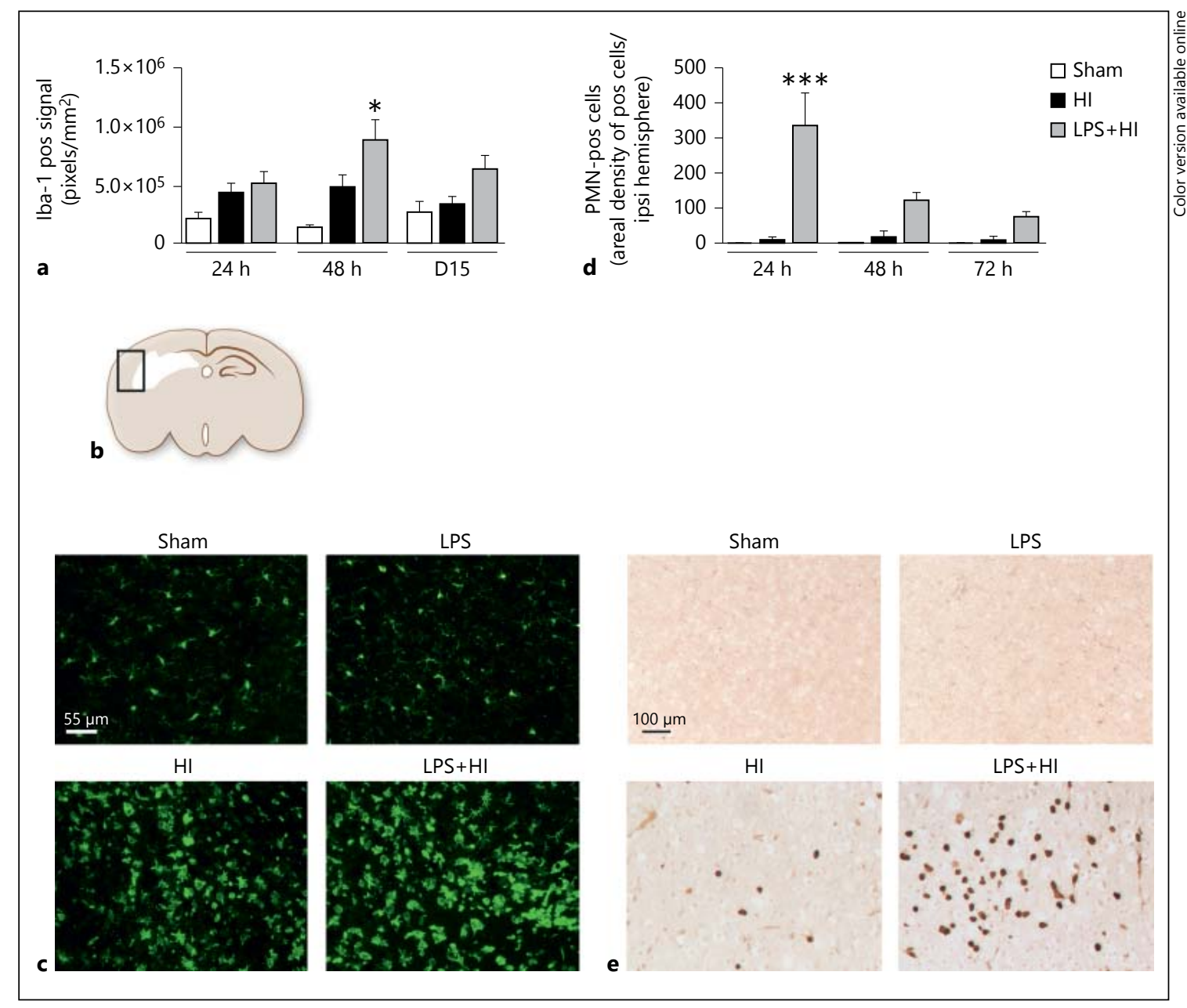

Fig. 6. Activation/influx of microglia/macrophages and neutrophils in/into the brain at different time points postinsult. The influx/activation of macrophages/microglia was measured by analyzing staining for Iba-1. Staining for PMN was used to assess the presence of neutrophils. a Quantification of Iba-1-positive signal in the ipsilateral cortical and hippocampal areas at hippocampal level in sham-operated (Sham), HI and LPS+HI mouse pups at D1, 2 and 15 postinsult. The mean value of the area of positive signal in 6 microscopic fields is shown. Two-way ANOVA: $\mathrm{F}_{(2,33)}=11.1$, $\mathrm{p}<0.001$. b Schematic coronal brain picture illustrating where photographs were taken in the damaged cortex. c Representative examples of Iba-1 staining within the infarcted area in the cortex

by MAP2 loss when compared to HI, but is also associated with a gradual increase of neuronal damage from $3 \mathrm{~h}$ up to D10 postinsult. In contrast, the total amount of MAP2 loss in HI animals had already developed early, at $3 \mathrm{~h}$ postinsult, without any further increase over time. Importantly, we observed a clear difference in the timing of acute neuronal damage (MAP2 loss) and actual loss of cerebral tissue, i.e. cyst formation after HI and LPS+HI. of sham-operated, LPS-alone (LPS), HI and LPS+HI animals at D2 postinsult. d Areal density of PMN-positive cells counted in the ipsilateral hemisphere in a coronal section at the hippocampal level of sham-operated, HI and LPS+HI mouse pups at D1, 2 and 3 postinsult. e Representative pictures of PMN staining in the (infarcted) cortex of sham-operated, LPS alone, HI and LPS+HI animals at D1 post-HI. Two-way ANOVA: $\mathrm{F}_{(2,35)}=25.3, \mathrm{p}<0.001$. Sham $\mathrm{n}=3-4$, HI $\mathrm{n}=5-7$, LPS + HI $\mathrm{n}=5-7$ per time point. Data are expressed as the mean \pm SEM. Data were analyzed using twoway ANOVA with Bonferroni post hoc tests. ${ }^{*} \mathrm{p}<0.05,{ }^{* * *} \mathrm{p}<$ 0.001. Pos $=$ Positive; ipsi $=$ ipsilateral.

Whereas MAP2 loss was already present at $3 \mathrm{~h}$ postinsult, our data show that volume loss of cerebral tissue does not start before D2-3 postinsult as shown by HE staining. So, acute neuronal damage measured by MAP2 loss is an early indicator of gray matter damage but is not one-to-one related to direct loss of cerebral tissue, which is delayed for about 2-3 days. Notably, the relatively mild HI insult induced microscopic loss of hippocampal architecture 
and some loss of cortical volume but did not induce a macroscopically detectable cystic lesion. However, when HI was preceded by LPS, a major cystic lesion developed starting at D3-4, which increased until D10 postinsult. These data are important because they suggest that the time window for promising pharmacological interventions after HI probably changes when $\mathrm{HI}$ is preceded or combined with inflammation. We recently described a treatment strategy to regenerate $\mathrm{HI}$ brain injury by administering mesenchymal stem cells $[42,43]$. An advantage of this neuroregenerative strategy is a long therapeutic window; treatment with mesenchymal stem cells leads to repair of the lesion, even when started 10 days after HI. However, since the timing of the 'damage response' to HI changes when $\mathrm{HI}$ is accompanied by inflammation, one should take into account that inflammation may considerably shorten the time window of MSC treatment. In particular, the formation of acellular cysts that develop from 3-4 days after LPS+HI might require acceleration of the start of regenerative therapies. Our data may also guide other therapeutic regenerative or neuroprotective strategies currently being investigated for neonatal encephalopathy, like erythropoietin or melatonin treatment [44-47].

The influence of combined exposure to inflammation and HI on cerebral white matter injury was measured by loss of MBP staining in the infarct area. As described before, LPS induced a significant increase in HI-induced cerebral white matter damage [37]. Here we show that LPS $+\mathrm{HI}$ also affected myelination in white matter structures outside the infarcted area, which can have important consequences for neurodevelopmental outcome. LPS+HI induced thinning of the corpus callosum, an important white matter structure that facilitates communication between both hemispheres. The thickness of the corpus callosum can be influenced by a deficit in myelination and/or axonal damage. In human neonates, a reduction in corpus callosum thickness is associated with cognitive and motor impairment [48-50]. Additionally, arborization of the myelinated fibers in the cingulum was strongly reduced by LPS+HI. Axonal injury in this part of the brain has been shown to be involved in cognition, working-memory performance and motor function, for example [23, 48, 51, 52].

The observed effects of LPS+HI on myelination urged us to investigate the effect of LPS+HI on oligodendrocytes [53]. Interestingly, after $\mathrm{HI}$, the areal density of Olig2-positive cells was upregulated selectively in the infarcted area. We have shown that sensitization by LPS led to an even stronger upregulation of Olig2-positive cells in the infarcted area after HI. In line with a decrease in MBP staining, CNPase-positive signal, as a marker for more mature myelinating oligodendrocytes, was strongly decreased in the infarcted area after HI and was almost completely absent after LPS + HI. Our data might indicate that $\mathrm{HI}$ and LPS+HI induces oligodendrogliogenesis or migration of oligodendrocytes to the site of damage in an attempt to repair myelination of the white matter tracts. However, since there is a decrease in more mature myelinating oligodendrocytes, the development of oligodendrocyte precursors into the final mature myelinating stage might be delayed or even arrested after LPS+HI. In line with our data, previous animal studies have shown that neonatal HI or systemic inflammation result in proliferation of oligodendrocytes, which is often followed by an arrest in differentiation and maturation into mature oligodendrocytes [54, 55]. Importantly, this phenomenon is also described in human neonates following periventricular leukomalacia $[29,56,57]$. An arrest in oligodendrocyte maturation will eventually result in a decrease in myelin production, white matter damage and axonal injury.

A proinflammatory state during the perinatal period can already affect oligodendrocyte precursors and elicit cerebral white matter damage and behavioral deficits, as shown in animal models [58-64]. However, in a control group we injected LPS $(0.5 \mathrm{mg} / \mathrm{kg})$ intraperitoneally without induction of HI, but we did not observe any aberrations in cerebral gray or white matter structures or tissue loss in our neonatal mouse model. The formation of free radicals, excitotoxicity, astrogliosis and release of cytokines by activated microglia are important downstream mechanisms that can damage the developing oligodendrocyte after inflammation and/or HI $[53,65,66]$. In particular, TNF- $\alpha$ has been described to negatively influencing oligodendrocyte maturation and/or induce oligodendrocyte death [53, 67-69]. Apparently, the changes induced by LPS alone are not sufficient to induce white or gray matter damage in our mouse model. LPS injection prior to $\mathrm{HI}$ resulted in an increased expression of all measured proinflammatory cytokines and chemokines in the ipsilateral hemisphere compared to HI. Besides the increased upregulation, levels of TNF- $\alpha$, TGF- $\beta$, IL- 10 and MCP-1 were elevated for a prolonged time up to D2-3. Particularly TNF- $\alpha$ expression was strongly increased and prolonged following LPS $+\mathrm{HI}$ and is likely a crucial factor contributing to the arrest in oligodendrocyte maturation $[53,67,68,70]$. We showed earlier that $\mathrm{HI}$-induced changes in cytokine mRNA levels were related to changes in the protein levels of these cytokines [71]. Besides the effect on
Bonestroo/Heijnen/Groenendaal/van Bel/ Nijboer 
oligodendrocytes, Kendall et al. [16] showed that TNF- $\alpha$ is a key factor in the LPS-mediated sensitizing effect on HI brain damage, since deletion of the TNF gene cluster prevented the LPS-induced increase in endothelial and microglia activation and the increase in HI brain damage. Consequently, anti-TNF therapies could be promising in neonates in whom inflammation precedes $\mathrm{HI}$.

Besides the increased upregulation of proinflammatory cytokines, we also observed a strong increase in antiinflammatory TGF- $\beta$ and IL-10 following LPS+HI. TGF- $\beta$ was hardly upregulated after HI alone, but LPS injection induced elevated and prolonged expression from D2-3. Besides possible anti-inflammatory effects of TGF- $\beta$, Nobuta et al. [72] showed that TGF- $\beta$ is also an important inhibitor of oligodendrocyte proliferation and differentiation in vitro and that TGF- $\beta$ is extensively expressed in the white matter following cerebral white matter injury in human neonates. We suggest that the strong and early rise in TNF- $\alpha$, for example, might have an early damaging effect on oligodendrocytes and result in an increase in oligodendrocyte proliferation (i.e. an increased areal density of Olig2-positive cells), followed by a possible second hit on oligodendrocyte maturation caused by increased TGF- $\beta$ expression at D1-3. TGF- $\beta$ signaling could therefore also be an interesting target to reduce white matter injury following LPS+HI.

LPS $+\mathrm{HI}$ induced an increase in IL-10 mRNA expression at D1 compared to HI only. IL-10 is an anti-inflammatory cytokine capable of suppressing proinflammatory cytokine production and has been shown to have a protective effect on developing white matter [73-75]. However, our present data indicate that the possible protective effect of IL-10 might become overshadowed by the strong proinflammatory response induced by LPS.

Besides the effects of damaging signals like free radical formation, excitotoxicity or cytokine release, maturation and survival of oligodendrocytes is also dependent on the availability of several growth and trophic factors secreted by local cells like microglia, astrocytes and neurons, including insulin-like growth factors (IGFs), nerve growth factor, ciliary-neurotrophic factor (CNTF), platelet-derived growth factor and fibroblast growth factor [76, 77]. Pang et al. [68] showed that LPS causes a reduction in microglia-derived growth factors IGF-1 and CNTF, which was associated with oligodendrocyte death in vitro. However, the exact effects of LPS+HI on these growth factors in vivo are still unknown. We feel that ongoing research should also aim at further unraveling the effect of inflammation on trophic and growth factors involved in the differentiation and maturation of oligodendro-

Brain Injury after Inflammatory Perinatal Asphyxia cytes, so that future therapies can circumvent the arrest in oligodendrocyte maturation to prevent white matter injury in the context of perinatal inflammation.

Microglial activation results in the release of harmful molecules, including nitric oxide, glutamate and cytokines, which can aggravate brain damage. Several studies have shown that microglia play an important role in the sensitizing effect of LPS on HI brain damage [37, 68, 78, 79]. In this study we observed an increased Iba-1-positive signal and morphologically an increased activation state of microglia/macrophages in the brain 2 days following LPS+HI when compared to HI only. The increased Iba1-positive signal is probably caused by infiltration of peripheral macrophages to the site of injury. Interestingly, at D1 after insult, no significant difference in Iba-1 staining was observed between HI and LPS+HI animals. It could be that LPS induces priming of microglia in an earlier phase without clear morphological changes assessed by Iba-1 staining [37].

LPS+HI caused a dramatic increase in CINC-1 expression and neutrophil influx into the brain within 1 day postinsult when compared to HI. Besides the increased CINC-1 expression, the early and rapid influx of neutrophils might be explained by the influence of systemic inflammation on adhesion molecule expression on endothelial cells of the BBB, leading to more directed trafficking of immune cells to the lesion site [17]. Furthermore, the increase in peripheral cytokines following systemic LPS may influence signaling cascades in leukocytes, thereby enhancing their migratory potential [80, 81]. Neutrophils play an important role in clearing cellular debris after tissue damage. However, after activation they release cytotoxic molecules that can damage surrounding tissue. We and others have previously shown that neutrophil depletion can reduce HI-induced brain damage [8284]. Prevention of the early influx of neutrophils, by inhibiting chemotactic cues like CINC-1 in the periphery or brain, could be an interesting therapeutic option to prevent the exacerbation of HI brain injury after LPS+HI.

In conclusion, our data show that when a $\mathrm{HI}$ insult is preceded by an inflammatory stimulus the timing of brain injury changes and additional molecular and/or cellular pathways contribute to the evolvement of the lesion. These changes in timing and pathways are important because they might require modifications in the time window, dosage or a combination of promising experimental therapies to result in effective and successful neuroprotection or neuroregeneration after inflammatory perinatal asphyxia. Hypothermia is currently the only standardized treatment for full-term neonates suffering 
from an $\mathrm{HI}$ insult. It is important to realize that exacerbation and prolongation of the 'damage response' induced by inflammation can have important implications for the efficacy of hypothermia treatment after HI. Hypothermia has been shown to be only effective when started within $6 \mathrm{~h}$ after $\mathrm{HI}$ in term neonates $[85,86]$. Recently, Osredkar et al. [87] showed that hypothermia has no neuroprotective effect after LPS-sensitized HI brain injury. Furthermore, Wintermark et al. [9] suggested that chorioamnionitis with fetal vasculitis may limit the effectiveness of therapeutic hypothermia following $\mathrm{HI}$ in human full-term neonates. If the metabolic state of the brain is increased or altered due to (preceding) inflammation, cooling might be less effective. The loss of effec- tiveness of the only clinically applied therapy at present in term neonates suffering from inflammatory perinatal asphyxia further highlights the indispensable need to explore novel therapies specifically aimed at dampening the inflammatory component. Moreover, current treatments using anti-inflammatory strategies may have to be continued for a longer period, and/or have to be intensified to suppress the increased and prolonged neuroinflammatory response.

\section{Disclosure Statement}

No duality of interest to declare.

\section{References}

1 Perlman M, Shah PS: Hypoxic-ischemic encephalopathy: challenges in outcome and prediction. J Pediatr 2011;158:e51-e54.

2 Ferriero DM: Neonatal brain injury. N Engl J Med 2004;351:1985-1995.

3 Groenendaal F, Casaer A, Dijkman KP, Gavilanes AW, de Haan TR, ter Horst HJ, Laroche S, Naulaers G, Rijken M, van Straaten HL, Steiner K, Swarte RM, Zecic A, Zonnenberg IA: Introduction of hypothermia for neonates with perinatal asphyxia in the Netherlands and Flanders. Neonatology 2013;104:15-21.

-4 Grether JK, Nelson KB: Maternal infection and cerebral palsy in infants of normal birth weight. JAMA 1997;278:207-211.

5 Impey L, Greenwood C, MacQuillan K, Reynolds M, Sheil O: Fever in labour and neonatal encephalopathy: a prospective cohort study. BJOG 2001;108:594-597.

-6 Blume HK, Li CI, Loch CM, Koepsell TD: Intrapartum fever and chorioamnionitis as risks for encephalopathy in term newborns: a casecontrol study. Dev Med Child Neurol 2008; 50:19-24.

7 Hayes BC, Cooley S, Donnelly J, Doherty E, Grehan A, Madigan C, McGarvey C, Mulvany S, Ryan S, Gillian J, Geary MP, Matthews TG, King MD: The placenta in infants $>36$ weeks gestation with neonatal encephalopathy: a case control study. Arch Dis Child Fetal Neonatal Ed 2013;98:F233-F239.

8 Peebles DM, Wyatt JS: Synergy between antenatal exposure to infection and intrapartum events in causation of perinatal brain injury at term. BJOG 2002;109:737-739.

$\$$ Wintermark P, Boyd T, Gregas MC, Labrecque M, Hansen A: Placental pathology in asphyxiated newborns meeting the criteria for therapeutic hypothermia. Am J Obstet Gynecol 2010;203:579.e1-e9.

10 Harteman JC, Nikkels PG, Benders MJ, Kwee A, Groenendaal F, de Vries LS: Placental pa- thology in full-term infants with hypoxicischemic neonatal encephalopathy and association with magnetic resonance imaging pattern of brain injury. J Pediatr 2013;163: 968-995.e2.

11 Shalak LF, Laptook AR, Jafri HS, Ramilo O, Perlman JM: Clinical chorioamnionitis, elevated cytokines, and brain injury in term infants. Pediatrics 2002;110:673-680.

12 Akira S, Uematsu S, Takeuchi O: Pathogen recognition and innate immunity. Cell 2006; 124:783-801.

13 Wang X, Rousset CI, Hagberg H, Mallard C: Lipopolysaccharide-induced inflammation and perinatal brain injury. Semin Fetal Neonatal Med 2006;11:343-353.

14 Rice JE 3rd, Vannucci RC, Brierley JB: The influence of immaturity on hypoxic-ischemic brain damage in the rat. Ann Neurol 1981;9: 131-141.

15 Eklind S, Mallard C, Leverin AL, Gilland E, Blomgren K, Mattsby-Baltzer I, Hagberg H: Bacterial endotoxin sensitizes the immature brain to hypoxic-ischaemic injury. Eur J Neurosci 2001;13:1101-1106.

16 Kendall GS, Hristova M, Horn S, Dafou D, Acosta-Saltos A, Almolda B, Zbarsky V, Rumajogee P, Heuer H, Castellano B, Pfeffer K, Nedospasov SA, Peebles DM, Raivich G: TNF gene cluster deletion abolishes lipopolysaccharide-mediated sensitization of the neonatal brain to hypoxic ischemic insult. Lab Invest 2011;91:328-341.

17 Mallard C: Innate immune regulation by tolllike receptors in the brain. ISRN Neurol 2012; 2012:701950.

18 Coumans AB, Middelanis JS, Garnier Y, Vaihinger HM, Leib SL, von Duering MU, Hasaart TH, Jensen A, Berger R: Intracisternal application of endotoxin enhances the susceptibility to subsequent hypoxic-ischemic brain damage in neonatal rats. Pediatr Res 2003;53: 770-775.
19 Eklind S, Mallard C, Arvidsson P, Hagberg H: Lipopolysaccharide induces both a primary and a secondary phase of sensitization in the developing rat brain. Pediatr Res 2005;58: 112-116.

20 Wang X, Hagberg H, Nie C, Zhu C, Ikeda T, Mallard C: Dual role of intrauterine immune challenge on neonatal and adult brain vulnerability to hypoxia-ischemia. J Neuropathol Exp Neurol 2007;66:552-561.

21 Yang L, Sameshima H, Ikeda T, Ikenoue T: Lipopolysaccharide administration enhances hypoxic-ischemic brain damage in newborn rats. J Obstet Gynaecol Res 2004;30: 142-147.

22 Filgueiras CC, Manhaes AC: Increased lateralization in rotational side preference in male mice rendered acallosal by prenatal gamma irradiation. Behav Brain Res 2005;162:289298.

23 van Velthoven CT, van de Looij Y, Kavelaars A, Zijlstra J, van Bel F, Huppi PS, Sizonenko S, Heijnen CJ: Mesenchymal stem cells restore cortical rewiring after neonatal ischemia in mice. Ann Neurol 2012;71:785-796.

24 Ito D, Imai Y, Ohsawa K, Nakajima K, Fukuuchi Y, Kohsaka S: Microglia-specific localisation of a novel calcium binding protein, Iba1. Brain Res Mol Brain Res 1998;57: $1-9$.

25 Sasaki Y, Ohsawa K, Kanazawa, Kohsaka S, Imai Y: Ibal is an actin-cross-linking protein in macrophages/microglia. Biochem Biophys Res Commun 2001;286:292-297.

26 Ito D, Tanaka K, Suzuki S, Dembo T, Fukuuchi Y: Enhanced expression of Ibal, ionized calcium-binding adapter molecule 1, after transient focal cerebral ischemia in rat brain. Stroke 2001;32:1208-1215.

27 Dammann O, Leviton A: Inflammatory brain damage in preterm newborns - dry numbers, wet lab, and causal inferences. Early Hum Dev 2004;79:1-15. 
28 Hagberg H, Gressens P, Mallard C: Inflammation during fetal and neonatal life: implications for neurologic and neuropsychiatric disease in children and adults. Ann Neurol 2012; 71:444-457.

29 Volpe JJ: Brain injury in premature infants: a complex amalgam of destructive and developmental disturbances. Lancet Neurol 2009; 8:110-124.

- 30 Leviton A, Paneth N, Reuss ML, Susser M, Allred EN, Dammann O, Kuban K, Van Marter LJ, Pagano M, Hegyi T, Hiatt M, Sanocka U, Shahrivar F, Abiri M, Disalvo D, Doubilet P, Kairam R, Kazam E, Kirpekar M, Rosenfeld D, Schonfeld S, Share J, Collins M, Genest D, Shen-Schwarz S: Maternal infection, fetal inflammatory response, and brain damage in very low birth weight infants. Pediatr Res 1999;46:566-575.

-31 Wu YW, Escobar GJ, Grether JK, Croen LA, Greene JD, Newman TB: Chorioamnionitis and cerebral palsy in term and near-term infants. JAMA 2003;290:2677-2684.

-32 Yoon BH, Park CW, Chaiworapongsa T: Intrauterine infection and the development of cerebral palsy. BJOG 2003;110(suppl 20): 124-127.

33 Ratnayake U, Quinn T, LaRosa DA, Dickinson $\mathrm{H}$, Walker DW: Prenatal exposure to the viral mimetic poly I:C alters fetal brain cytokine expression and postnatal behaviour. Dev Neurosci 2014;36:83-94.

- 34 Bergeron JD, Deslauriers J, Grignon S, Fortier LC, Lepage M, Stroh T, Poyart C, Sébire G: White matter injury and autistic-like behavior predominantly affecting male rat offspring exposed to group B streptococcal maternal inflammation. Dev Neurosci 2013;35:504-515.

- 35 Balakrishnan B, Dai H, Janisse J, Romero R, Kannan S: Maternal endotoxin exposure results in abnormal neuronal architecture in the newborn rabbit. Dev Neurosci 2013;35:396405.

-36 Eklind S, Hagberg H, Wang X, Savman K, Leverin AL, Hedtjarn M, Mallard C: Effect of lipopolysaccharide on global gene expression in the immature rat brain. Pediatr Res 2006; 60:161-168

-37 Wang X, Stridh L, Li W, Dean J, Elmgren A, Gan L, Eriksson K, Hagberg H, Mallard C: Lipopolysaccharide sensitizes neonatal hypoxicischemic brain injury in a MyD88-dependent manner. J Immunol 2009;183:7471-7477.

-38 Degos V, Peineau S, Nijboer C, Kaindl AM, Sigaut S, Favrais G, Plaisant F, Teissier N, Gouadon E, Lombet A, Saliba E, Collingridge GL, Maze M, Nicoletti F, Heijnen C, Mantz J, Kavelaars A, Gressens P: G protein-coupled receptor kinase 2 and group I metabotropic glutamate receptors mediate inflammationinduced sensitization to excitotoxic neurodegeneration. Ann Neurol 2013;73:667-678.

-39 Nijboer CH, Heijnen CJ, Degos V, Willemen HL, Gressens P, Kavelaars A: Astrocyte GRK2 as a novel regulator of glutamate transport and brain damage. Neurobiol Dis 2013;54: 206-215.
40 Singh AK, Jiang Y: How does peripheral lipopolysaccharide induce gene expression in the brain of rats? Toxicology 2004;201:197-207.

41 Xaio H, Banks WA, Niehoff ML, Morley JE: Effect of LPS on the permeability of the bloodbrain barrier to insulin. Brain Res 2001;896: 36-42.

42 Donega V, van Velthoven CT, Nijboer $\mathrm{CH}$, van Bel F, Kas MJ, Kavelaars A, Heijnen CJ: Intranasal mesenchymal stem cell treatment for neonatal brain damage: long-term cognitive and sensorimotor improvement. PLoS One 2013;8:e51253.

43 van Velthoven CT, Kavelaars A, van Bel F, Heijnen CJ: Mesenchymal stem cell treatment after neonatal hypoxic-ischemic brain injury improves behavioral outcome and induces neuronal and oligodendrocyte regeneration. Brain Behav Immun 2010;24:387-393.

44 Chang YS, Mu D, Wendland M, Sheldon RA, Vexler ZS, McQuillen PS, Ferriero DM: Erythropoietin improves functional and histological outcome in neonatal stroke. Pediatr Res 2005;58:106-111.

45 Juul SE, McPherson RJ, Bauer LA, Ledbetter KJ, Gleason CA, Mayock DE: A phase I/II trial of high-dose erythropoietin in extremely low birth weight infants: pharmacokinetics and safety. Pediatrics 2008;122:383-391.

46 Welin AK, Svedin P, Lapatto R, Sultan B, Hagberg H, Gressens P, Kjellmer I, Mallard C: Melatonin reduces inflammation and cell death in white matter in the mid-gestation fetal sheep following umbilical cord occlusion. Pediatr Res 2007;61:153-158.

-47 Fan X, Heijnen CJ, van der Kooij MA, Groenendaal F, van Bel F: Beneficial effect of erythropoietin on sensorimotor function and white matter after hypoxia-ischemia in neonatal mice. Pediatr Res 2011;69:56-61.

48 Tusor N, Wusthoff C, Smee N, Merchant N, Arichi T, Allsop JM, Cowan FM, Azzopardi D, Edwards AD, Counsell SJ: Prediction of neurodevelopmental outcome after hypoxicischemic encephalopathy treated with hypothermia by diffusion tensor imaging analyzed using tract-based spatial statistics. Pediatr Res 2012;72:63-69.

49 Caldu X, Narberhaus A, Junque C, Gimenez $M$, Vendrell P, Bargallo N, Segarra D, Botet F: Corpus callosum size and neuropsychologic impairment in adolescents who were born preterm. J Child Neurol 2006;21:406-410.

50 van Kooij BJ, van Handel M, Uiterwaal CS, Groenendaal F, Nievelstein RA, Rademaker KJ, Jongmans MJ, DE Vries LS: Corpus callosum size in relation to motor performance in 9- to 10-year-old children with neonatal encephalopathy. Pediatr Res 2008;63:103108.

51 van Kooij BJ, de Vries LS, Ball G, van Haastert IC, Benders MJ, Groenendaal F, Counsell SJ: Neonatal tract-based spatial statistics findings and outcome in preterm infants. AJNR Am J Neuroradiol 2012;33:188-194.

52 Ewing-Cobbs L, Prasad MR, Swank P, Kramer L, Cox CS Jr, Fletcher JM, Barnes M, Zhang
X, Hasan KM: Arrested development and disrupted callosal microstructure following pediatric traumatic brain injury: relation to neurobehavioral outcomes. Neuroimage 2008;42: 1305-1315.

53 Volpe JJ, Kinney HC, Jensen FE, Rosenberg PA: The developing oligodendrocyte: key cellular target in brain injury in the premature infant. Int J Dev Neurosci 2011;29:423440

54 Back SA, Han BH, Luo NL, Chricton CA, Xanthoudakis S, Tam J, Arvin KL, Holtzman DM: Selective vulnerability of late oligodendrocyte progenitors to hypoxia-ischemia. J Neurosci 2002;22:455-463.

55 Huang Z, Liu J, Cheung PY, Chen C: Longterm cognitive impairment and myelination deficiency in a rat model of perinatal hypoxicischemic brain injury. Brain Res 2009;1301: 100-109.

56 Buser JR, Maire J, Riddle A, Gong X, Nguyen T, Nelson K, Luo NL, Ren J, Struve J, Sherman LS, Miller SP, Chau V, Hendson G, Ballabh P, Grafe MR, Back SA: Arrested preoligodendrocyte maturation contributes to myelination failure in premature infants. Ann Neurol 2012;71:93-109.

57 Billiards SS, Haynes RL, Folkerth RD, Borenstein NS, Trachtenberg FL, Rowitch DH, Ligon KL, Volpe JJ, Kinney HC: Myelin abnormalities without oligodendrocyte loss in periventricular leukomalacia. Brain Pathol 2008; 18:153-163.

58 Dean JM, van de Looij Y, Sizonenko SV, Lodygensky GA, Lazeyras F, Bolouri $\mathrm{H}$, Kjellmer I, Huppi PS, Hagberg H, Mallard C: Delayed cortical impairment following lipopolysaccharide exposure in preterm fetal sheep. Ann Neurol 2011;70:846-856.

59 Hagberg H, Peebles D, Mallard C: Models of white matter injury: comparison of infectious, hypoxic-ischemic, and excitotoxic insults. Ment Retard Dev Disabil Res Rev 2002;8:3038.

60 Duncan JR, Cock ML, Scheerlinck JP, Westcott KT, McLean C, Harding R, Rees SM: White matter injury after repeated endotoxin exposure in the preterm ovine fetus. Pediatr Res 2002;52:941-949.

61 Garnier Y, Berger R, Alm S, von Duering MU, Coumans AB, Michetti F, Bruschettini M, Lituania M, Hasaart TH, Gazzolo D: Systemic endotoxin administration results in increased S100B protein blood levels and periventricular brain white matter injury in the preterm fetal sheep. Eur J Obstet Gynecol Reprod Biol 2006;124:15-22.

62 Mallard C, Welin AK, Peebles D, Hagberg H, Kjellmer I: White matter injury following systemic endotoxemia or asphyxia in the fetal sheep. Neurochem Res 2003;28:215-223.

63 Pang Y, Cai Z, Rhodes PG: Disturbance of oligodendrocyte development, hypomyelination and white matter injury in the neonatal rat brain after intracerebral injection of lipopolysaccharide. Brain Res Dev Brain Res 2003;140:205-214. 
64 Falahati S, Breu M, Waickman AT, Phillips AW, Arauz EJ, Snyder S, Porambo M, Goeral $\mathrm{K}$, Comi AM, Wilson MA, Johnston MV, Fatemi A: Ischemia-induced neuroinflammation is associated with disrupted development of oligodendrocyte progenitors in a model of periventricular leukomalacia. Dev Neurosci 2013;35:182-196.

65 Segovia KN, McClure M, Moravec M, Luo NL, Wan Y, Gong X, Riddle A, Craig A, Struve J, Sherman LS, Back SA: Arrested oligodendrocyte lineage maturation in chronic perinatal white matter injury. Ann Neurol 2008;63: 520-530.

66 Back SA, Tuohy TM, Chen H, Wallingford N, Craig A, Struve J, Luo NL, Banine F, Liu Y, Chang A, Trapp BD, Bebo BF Jr, Rao MS, Sherman LS: Hyaluronan accumulates in demyelinated lesions and inhibits oligodendrocyte progenitor maturation. Nat Med 2005; 11:966-972.

-67 Kadhim H, Tabarki B, Verellen G, de Prez C, Rona AM, Sebire G: Inflammatory cytokines in the pathogenesis of periventricular leukomalacia. Neurology 2001;56:1278-1284.

-68 Pang Y, Campbell L, Zheng B, Fan L, Cai Z, Rhodes P: Lipopolysaccharide-activated microglia induce death of oligodendrocyte progenitor cells and impede their development. Neuroscience 2010;166:464-475.

-69 Paintlia MK, Paintlia AS, Singh AK, Singh I: Synergistic activity of interleukin-17 and tumor necrosis factor- $\alpha$ enhances oxidative stress-mediated oligodendrocyte apoptosis. J Neurochem 2011;116:508-521.

-70 Selmaj K, Raine CS, Farooq M, Norton WT, Brosnan CF: Cytokine cytotoxicity against oligodendrocytes: apoptosis induced by lymphotoxin. J Immunol 1991;147:1522-1529.

71 Nijboer CH, Heijnen CJ, Groenendaal F, van Bel F, Kavelaars A: Alternate pathways preserve tumor necrosis factor- $\alpha$ production after nuclear factor- $\kappa B$ inhibition in neonatal cerebral hypoxia-ischemia. Stroke 2009;40: 3362-3368.
72 Nobuta H, Ghiani CA, Paez PM, Spreuer V, Dong H, Korsak RA, Manukyan A, Li J, Vinters HV, Huang EJ, Rowitch DH, Sofroniew MV, Campagnoni AT, de Vellis J, Waschek JA: STAT3-mediated astrogliosis protects myelin development in neonatal brain injury. Ann Neurol 2012;72:750-765.

73 Rodts-Palenik S, Wyatt-Ashmead J, Pang Y, Thigpen B, Cai Z, Rhodes P, Martin JN, Granger J, Bennett WA: Maternal infectioninduced white matter injury is reduced by treatment with interleukin-10. Am J Obstet Gynecol 2004;191:1387-1392.

-74 Brehmer F, Bendix I, Prager S, van de Looij Y, Reinboth BS, Zimmermanns J, Schlager GW, Brait D, Sifringer M, Endesfelder S, Sizonenko S, Mallard C, Buhrer C, Felderhoff-Mueser $\mathrm{U}$, Gerstner B: Interaction of inflammation and hyperoxia in a rat model of neonatal white matter damage. PLoS One 2012; 7:e49023.

75 Pang Y, Rodts-Palenik S, Cai Z, Bennett WA, Rhodes PG: Suppression of glial activation is involved in the protection of IL-10 on maternal $E$. coli induced neonatal white matter injury. Brain Res Dev Brain Res 2005;157:141149.

76 Barres BA, Schmid R, Sendnter M, Raff MC: Multiple extracellular signals are required for long-term oligodendrocyte survival. Development 1993;118:283-295.

77 Wolswijk G, Riddle PN, Noble M: Platelet-derived growth factor is mitogenic for O-2Aadult progenitor cells. Glia 1991;4:495-503.

78 Dean JM, Wang X, Kaindl AM, Gressens P, Fleiss B, Hagberg H, Mallard C: Microglial MyD88 signaling regulates acute neuronal toxicity of LPS-stimulated microglia in vitro. Brain Behav Immun 2010;24:776-783.

79 Lehnardt S, Massillon L, Follett P, Jensen FE, Ratan R, Rosenberg PA, Volpe JJ, Vartanian $\mathrm{T}$ : Activation of innate immunity in the CNS triggers neurodegeneration through a Tolllike receptor 4-dependent pathway. Proc Natl Acad Sci U S A 2003;100:8514-8519.
80 Vroon A, Heijnen CJ, Lombardi MS, Cobelens PM, Mayor F Jr, Caron MG, Kavelaars A: Reduced GRK2 level in T cells potentiates chemotaxis and signaling in response to CCL4. J Leukoc Biol 2004;75:901-909.

81 Vroon A, Kavelaars A, Limmroth V, Lombardi MS, Goebel MU, van Dam AM, Caron MG, Schedlowski M, Heijnen CJ: G proteincoupled receptor kinase 2 in multiple sclerosis and experimental autoimmune encephalomyelitis. J Immunol 2005; 174: 4400-4406.

82 Hudome S, Palmer C, Roberts RL, Mauger D, Housman C, Towfighi J: The role of neutrophils in the production of hypoxic-ischemic brain injury in the neonatal rat. Pediatr Res 1997;41:607-616.

83 Nijboer $\mathrm{CH}$, Kavelaars A, Vroon A, Groenendaal F, van Bel F, Heijnen CJ: Low endogenous G-protein-coupled receptor kinase 2 sensitizes the immature brain to hypoxia-ischemia-induced gray and white matter damage. J Neurosci 2008;28:33243332.

84 Palmer C, Roberts RL, Young PI: Timing of neutrophil depletion influences long-term neuroprotection in neonatal rat hypoxicischemic brain injury. Pediatr Res 2004;55: 549-556.

85 Azzopardi DV, Strohm B, Edwards AD, Dyet L, Halliday HL, Juszczak E, Kapellou O, Levene $M$, Marlow N, Porter E, Thoresen M, Whitelaw A, Brocklehurst P, TOBY Study Group: Moderate hypothermia to treat perinatal asphyxial encephalopathy. $\mathrm{N}$ Engl J Med 2009;361:1349-1358.

86 Johnston MV, Fatemi A, Wilson MA, Northington F: Treatment advances in neonatal neuroprotection and neurointensive care. Lancet Neurol 2011;10:372-382.

87 Osredkar D, Thoresen M, Maes E, Flatebo T, Elstad M, Sabir H: Hypothermia is not neuroprotective after infection-sensitized neonatal hypoxic-ischemic brain injury. Resuscitation 2014;85:567-572. 